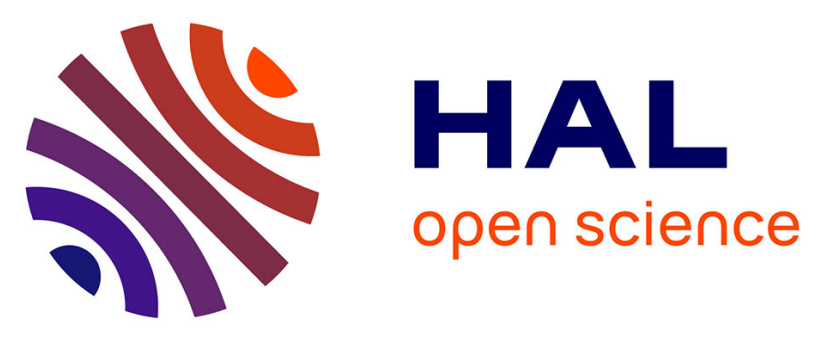

\title{
Synthesis of multi-hollow clay-armored latexes by surfactant-free emulsion polymerization of styrene mediated by poly(ethylene oxide)-based macroRAFT/Laponite complexes
}

Thiago Rodrigues Guimarães, Thaissa de Camargo Chaparro, Franck d'Agosto, Muriel Lansalot, Amilton Martins dos Santos, Elodie Bourgeat-Lami

\section{- To cite this version:}

Thiago Rodrigues Guimarães, Thaissa de Camargo Chaparro, Franck d'Agosto, Muriel Lansalot, Amilton Martins dos Santos, et al.. Synthesis of multi-hollow clay-armored latexes by surfactant-free emulsion polymerization of styrene mediated by poly(ethylene oxide)-based macroRAFT/Laponite complexes. Polymer Chemistry, 2014, 5 (22), pp.6611 - 6622. 10.1039/c4py00362d . hal-01859687

\section{HAL Id: hal-01859687 https://hal.science/hal-01859687}

Submitted on 13 Nov 2020

HAL is a multi-disciplinary open access archive for the deposit and dissemination of scientific research documents, whether they are published or not. The documents may come from teaching and research institutions in France or abroad, or from public or private research centers.
L'archive ouverte pluridisciplinaire HAL, est destinée au dépôt et à la diffusion de documents scientifiques de niveau recherche, publiés ou non, émanant des établissements d'enseignement et de recherche français ou étrangers, des laboratoires publics ou privés. 


\section{Synthesis of multi-hollow clay-armored latexes by surfactant-free}

$$
\text { emulsion polymerization of styrene mediated by }
$$

poly(ethylene oxide)-based macroRAFT/Laponite complexes.

Thiago Rodrigues Guimarães, ${ }^{1,2}$ Thaissa de Camargo Chaparro, ${ }^{2}$ Franck D'Agosto, ${ }^{1}$ Muriel Lansalot, ${ }^{1}$ Amilton Martins Dos Santos, ${ }^{2}$ Elodie Bourgeat-Lami ${ }^{1} *$

${ }^{1}$ Université de Lyon, Univ. Lyon 1, CPE Lyon, CNRS, UMR 5265, Laboratoire de Chimie, Catalyse, Polymères et Procédés (C2P2), LCPP group, 43, Bd. du 11 Novembre 1918, F69616 Villeurbanne, France.

${ }^{2}$ Laboratory of Polymer, Department of Chemical Engineering, Lorena Engineering School, University of São Paulo, Estrada Municipal do Campinho, S/N, 12.602.810, Lorena (SP), Brazil.

KEYWORDS. RAFT, nanocomposites, Laponite, emulsion polymerization, poly(ethylene oxide). 


\section{ABSTRACT}

We report the surfactant-free emulsion polymerization of styrene mediated by a trithiocarbonate poly(ethylene oxide)-based macromolecular RAFT agent (PEO2 $K$-TTC, $M_{\mathrm{n}}$ $\left.(\mathrm{PEO})=2000 \mathrm{~g} \mathrm{~mol}^{-1}\right)$ in presence of Laponite clay platelets. Adsorption studies revealed high affinity of the macroRAFT for Laponite. The resulting macroRAFT/clay complexes were used to control the growth of polystyrene chains and generate clay-armored latexes. Undesirable effects observed under the same conditions in the absence of clay, such as rate retardation, long induction period and loss of colloidal stability were not observed when the macroRAFT was immobilized on the clay surface. A minimum amount of macroRAFT was necessary to ensure a good control of the polymerization and a narrow molar mass distribution while a too large amount led to similar effects as in the absence of clay due to partitioning of the free non-adsorbing PEO2K-TTC macroRAFT. By a careful selection of the macroRAFT and monomer concentrations, stable multi-hollow clay-armored composite latex particles containing embedded PEO and composed of polymer chains of controlled and narrowly distributed molar masses could be successfully obtained by this technique. 


\section{INTRODUCTION}

The ability to control macromolecular architectures is of key importance in designing novel materials with improved properties. Since the pioneering works on reversible-deactivation radical polymerization (RDRP), ${ }^{1,2}$ an increasing number of studies have been reported. The benefits of RDRP techniques for the development of new polymers or copolymers with predetermined structures and functionalities have revolutionized the macromolecular design. These techniques require the use of organic molecules acting as controlling agents during the polymerization. Well-defined polymer chains of predetermined molar mass can thus be synthesized. On the other hand, organic/inorganic nanocomposite materials have attracted increasing attention over the last years, owing to their increased mechanical, electrical, thermal or barrier properties over pure polymeric materials. ${ }^{3}$ In this respect, RDRP has proven to be a versatile and robust method to introduce a variety of well-defined organic polymers and copolymers on inorganic particles or substrates. Most effective strategies in this field rely on an easy functionalization of the inorganic with controlling agents for further growth of densely grafted polymer brushes in organic ${ }^{4,5,6,7,8,9}$ or aqueous solutions. ${ }^{10,11,12} \mathrm{~A}$ wide range of organic/inorganic hybrid materials have been prepared by these methods opening up new perspectives for the development of functional nanostructured materials that could find applications in a variety of domains such as surface patterning, electro-optical devices, biology or reinforced coatings. ${ }^{13,14}$ However, to date comparatively much less attention has been paid to the elaboration of organic/inorganic particles through polymerization of hydrophobic monomers in aqueous dispersed media using RDRP techniques. ${ }^{15}$ Indeed, the implementation of RDRP processes in aqueous dispersed media has long suffered from the complexities of particle nucleation and growth inherent to such systems, ${ }^{16}$ which has inevitably delayed further developments and in particular their application to the elaboration of composite colloids. 
Among the various RDRP processes, reversible addition-fragmentation chain transfer (RAFT) is receiving a lot of attention. ${ }^{17,18,19,20}$ One major difficulty in performing RAFTmediated emulsion polymerization is, however, that most RAFT agents are slightly hydrophobic and can therefore enter monomer droplets leading to transport issues from the droplets to the polymerization sites. This results in a poor colloidal stability, low polymerization rates and a poor control of the molar mass due to the formation of multipopulated polymer chains having grown in different environments (i.e., a broad molar mass distribution). ${ }^{21,22,23}$ One efficient way to overcome such difficulties has been first implemented by Ferguson et al. ${ }^{24}$ who used water-soluble amphipathic macromolecular RAFT agents capable to initiate the growth of hydrophobic blocks under starved monomer conditions leading to the in situ formation of block copolymers that self-assemble into latex particles in the absence of molecular surfactant. This polymerization-induced self-assembly (PISA) strategy was further expanded to batch processes using various hydrophilic macroRAFT agents including PEO-based macroRAFT agents. ${ }^{25,26,27,28,29}$ In these particular cases, the chemical nature and the solubility of the macroRAFT proved to be determinant in ensuring an efficient nucleation and the stabilization of the latex particles formed during the polymerization, the resulting particles sometimes exhibiting multiple nanosize domains (multi-hollow particles) due to the entrapment of PEO segments illustrating the complexity of the nucleation mechanism in these systems.

As mentioned above, only few works report the implementation of the RAFT process in the elaboration of organic/inorganic composite colloids. In 2008, Hartmann et al. ${ }^{30}$ described the polymer encapsulation of Montmorillonite clay platelets through miniemulsion polymerization using an intercalated cationic RAFT agent while Hawkett and coworkers ${ }^{31}$ reported for the first time the encapsulation of inorganic particles by RAFT-mediated emulsion polymerization using a strategy inspired from the self-assembly method described 
above. In Hawkett's strategy, amphipathic macroRAFT agents are first adsorbed on the surface of the inorganic particles. Their thiocarbonylthio extremity is further reactivated for the polymerization of a hydrophobic monomer under starved-feed emulsion polymerization conditions giving rise to the formation of an encapsulating hydrophobic shell. The macroRAFT agent being amphipathic, it is also capable to stabilize the resulting core-shell particles. This strategy, first developed for the encapsulation of titanium dioxide $\left(\mathrm{TiO}_{2}\right)$ pigments, ${ }^{31}$ was further extended to the encapsulation of gibbsite clay platelets, ${ }^{32}$ carbon nanotubes ${ }^{33,34}$ and cerium dioxide ${ }^{35}$ particles. However, these articles mainly focused on controlling particle morphology and little attention was paid to the livingness of the polymerization reaction with the exception of the preliminary papers of Hartmann ${ }^{30}$ and Hawkett. $^{31 \mathrm{a}, 32}$

Therefore, considering the very few number of studies dedicated to clay and the absence of literature on the design of polymer/Laponite nanocomposites through RDRP, we launched a study on the RAFT-mediated synthesis of polymer/Laponite particles. For that purpose, we selected a PEO macroRAFT agent bearing a trithiocarbonate end group (PEO2 $K$-TTC, $M_{\mathrm{n}}$ $\left.(\mathrm{PEO})=2000 \mathrm{~g} \mathrm{~mol}^{-1}\right)$. PEO oligomers are indeed known to have a significant affinity for clay surfaces, ${ }^{36}$ which should contribute to the localization of the polymerization at the clay/water interface and minimize macroRAFT partitioning between the monomer droplets and the aqueous phase. The resulting immobilization of the trithiocarbonate group onto Laponite will be then exploited to polymerize hydrophobic monomers. Particular attention will be paid to the resulting latex morphology and living features of the polymerization reaction. 


\section{EXPERIMENTAL SECTION}

Materials. Laponite is a trioctahedral synthetic hectorite manufactured by Rockwood Additives Ltd. (UK) as discoid platelets with a thickness of around $1 \mathrm{~nm}$, a diameter of about $30 \mathrm{~nm}$, a cation exchange capacity of $0.75 \mathrm{meq} \mathrm{g}^{-1}$, a specific surface area of $370 \mathrm{~m}^{2} \mathrm{~g}^{-1}$ and a negative surface charge density of $0.014 \mathrm{e}^{-} \AA^{-2} .{ }^{37}$ The clay used in this work was Laponite RD which is a fast-dispersing gel-grade without any added peptizer. The peptizer (tetrasodium pyrophosphate, $\mathrm{Na}_{4} \mathrm{P}_{2} \mathrm{O}_{7}, 95 \%$, Aldrich) was subsequently introduced in controlled amounts (10 wt $\%$ based on clay). Styrene (Sty, 99\%, Acros) was passed over an alumina column to remove the inhibitor before use. Methyl methacrylate (MMA, 99\%, Aldrich) and n-butyl acrylate (BA, 99\%, Aldrich), 4,4'-azobis(4-cyanopentanoic acid) (ACPA, 98\%, Fluka), anhydrous dichloromethane $\left(\mathrm{CH}_{2} \mathrm{Cl}_{2}, 99.8 \%\right.$, Aldrich), 4-(dimethylamino)pyridine (DMAP, 99\%, Aldrich), N,N'-dicyclohexylcarbodiimide (DCC, 99\%, Fluka), tetrahydrofuran (THF, Aldrich) and toluene (Aldrich) were all used as received. Poly(ethylene oxide) methyl ether (PEO2K, $2000 \mathrm{~g} \mathrm{~mol}^{-1}$, 99.5\%, Fluka) was dried by azeotropic distillation with $200 \mathrm{~mL}$ of toluene at $40{ }^{\circ} \mathrm{C}$. The 4-cyano-4-thiothiopropylsulfanyl pentanoic acid (CTPPA) RAFT agent was synthesized by the reaction of ACPA with bis(propyl-sulfanylthiocarbonyl) disulfide as reported elsewhere. ${ }^{38,39}{ }^{1} \mathrm{H}$ NMR $\left(\mathrm{CDCl}_{3}\right): \delta(\mathrm{ppm}) 1.03\left(\mathrm{t}, 3 \mathrm{H}, \mathrm{CH}_{3^{-}} \mathrm{CH}_{2^{-}}\right) ; 1.74(\mathrm{~m}, 2 \mathrm{H}$, $\left.\mathrm{CH}_{3}-\mathrm{CH}_{2}-\mathrm{CH}_{2}-\right) ; 1.89$ (s, 3H, $\left.-\mathrm{CH}_{2}-\mathrm{C}(\mathrm{CN})\left(\mathrm{CH}_{3}\right)-\mathrm{S}-\right) ; 2.34 \sim 2.67$ (m, 4H, -OOC-CH $\mathbf{C}_{2}-\mathrm{CH}_{2^{-}}$ $\left.\mathrm{C}(\mathrm{CN})\left(\mathrm{CH}_{3}\right)-\right) ; 3.31$ (t, 2H, $\left.-\mathrm{CH}_{2}-\mathbf{C H}_{2}-\mathrm{S}-\right)$. Yield: 62\%. Purity: 98\%. Deionized water (Purelab Classic UV, Elga LabWater) was used for all experiments.

Synthesis of the PEO2K-TTC macroRAFT agent. The poly(ethylene oxide) trithio carbonate macroRAFT agent (PEO2 $K$-TTC) was synthesized via an esterification reaction between PEO2 $K$ and CTPPA according to the literature. ${ }^{38}$ 
PEO2K-TTC adsorption isotherm. A Laponite stock dispersion $\left(20 \mathrm{~g} \mathrm{~L}^{-1}\right)$ was prepared by mixing $3.6 \mathrm{~g}$ of Laponite with $180 \mathrm{~mL}$ of an aqueous solution of tetrasodium pyrophosphate $\left(1 \mathrm{~g} \mathrm{~L}^{-1}\right)$ under stirring until a transparent suspension was obtained (ca. 30 min). A PEO2K-TTC stock solution $\left(40 \mathrm{~g} \mathrm{~L}^{-1}\right)$ was prepared by dissolving the macroRAFT agent in water until a homogeneous solution was obtained. Then, predetermined amounts of the PEO2K-TTC stock solution and water were added to a fixed amount of the Laponite suspension to cover a final PEO2K-TTC concentration range between 1 and $20 \mathrm{~g} \mathrm{~L}^{-1}$ while maintaining a fixed Laponite concentration of $10 \mathrm{~g} \mathrm{~L}^{-1}$. The dispersion was stirred for one hour and further ultracentrifuged at $60000 \mathrm{rpm}$ for one hour (Beckman Coulter Allegra 64R centrifuge). The equilibrium PEO2K-TTC concentration in the supernatant was determined by UV-visible spectroscopy (Jasco Instrument, Model V-530, France) using a pre-established calibration curve $\left(\lambda_{\max }=310 \mathrm{~nm}\right)$. The adsorbed amount, $Q e\left(\mathrm{mg} \mathrm{g}^{-1}\right)$, was then determined by the difference between the initial and the equilibrium concentrations according to:

$$
\mathrm{Q}_{\mathrm{e}}\left(\mathrm{mg} \mathrm{g}^{-1}\right)=\frac{\left(\mathrm{C}_{0}-\mathrm{C}_{\mathrm{e}}\right) \mathrm{V}}{\mathrm{m}} \times 1000
$$

where $\mathrm{C}_{0}\left(\mathrm{~g} \mathrm{~L}^{-1}\right)$ is the initial PEO2K-TTC concentration, Ce $\left(\mathrm{g} \mathrm{L}^{-1}\right)$ designates the PEO2KTTC equilibrium concentration in the supernatant, $\mathrm{V}(\mathrm{L})$ is the volume of solution and $\mathrm{m}(\mathrm{g})$ is the mass of Laponite.

Emulsion polymerization. In a typical surfactant-free emulsion polymerization experiment (run 2, Table 1), Laponite $(0.13 \mathrm{~g})$ was dispersed in $10 \mathrm{~mL}$ of water and stirred for $30 \mathrm{~min}$. Then, $1.5 \mathrm{~mL}$ of an aqueous solution of PEO2 $K$-TTC was added and the mixture was stirred for another $60 \mathrm{~min}$. The resulting dispersion was introduced in a $25 \mathrm{~mL}$ three-necked round bottle flask equipped with a condenser, a nitrogen purging tube and a magnetic stirrer. Then, styrene $\left(2.6 \mathrm{~g}, 1.9 \mathrm{~mol} \mathrm{~L}^{-1}\right)$ was introduced in the reactor and the suspension was 
deoxygenated under nitrogen for $30 \mathrm{~min}$ while heating to $70{ }^{\circ} \mathrm{C}$. ACPA $(0.013 \mathrm{~g})$ was dissolved in $1.5 \mathrm{~mL}$ of an aqueous solution of the peptizer $\left(9 \mathrm{~g} \mathrm{~L}^{-1}\right)$, and purged during 15 min under nitrogen. Adding this solution in the reaction medium started the polymerization, which was carried out for $24 \mathrm{~h}$. Samples were periodically withdrawn to follow monomer conversion as a function of time and the evolution of the molar mass and the molar mass distribution as a function of monomer conversion. The theoretical number-average molar mass, $M_{\mathrm{n} \text {, theo }}\left(\mathrm{g} \mathrm{mol}^{-1}\right)$, was determined neglecting the contribution of chains derived from the initiator using the following equation:

$$
\mathrm{M}_{\mathrm{n}, \text { theo }}=\mathrm{MM}_{\mathrm{PEO} 2 K-\mathrm{TTC}}+\frac{\mathrm{X}[\mathrm{Sty}] \mathrm{MM}_{\mathrm{Sty}}}{100[\mathrm{PEO} 2 K-\mathrm{TTC}]}
$$

where $\mathrm{X}(\%)$ is the monomer conversion, $[\mathrm{Sty}]\left(\mathrm{mol} \mathrm{L}^{-1}\right), \mathrm{MM}_{\text {Sty }}\left(\mathrm{g} \mathrm{mol}^{-1}\right)$ and $[\mathrm{PEO} 2 K$-TTC] $\left(\mathrm{mol} \mathrm{L}{ }^{-1}\right), \mathrm{MM}_{\mathrm{PEO} K \text {-TTC }}\left(\mathrm{g} \mathrm{mol}^{-1}\right)$ are the concentrations and molar masses of styrene and PEO2K-TTC, respectively.

Two semi-continuous emulsion polymerization reactions were also carried out at $70{ }^{\circ} \mathrm{C}$ for styrene and at $80{ }^{\circ} \mathrm{C}$ for MMA/BA (80:20 v/v) for 9 and 24 hours, respectively. The initial amount of monomer(s) was $5 \mathrm{wt} \%$ based on the overall monomer mass. The residual monomer(s) was added during 4 hours at the rate of 11.5 and $8.2 \mathrm{mg} \mathrm{min}^{-1}$, for styrene and MMA/BA, respectively. The polymerization was let to proceed for additional 5 and 19 hours for styrene and MMA/BA, respectively.

Polymer chains recovery for SEC analysis. The Laponite/polymer nanocomposite particles $(50 \mathrm{mg})$ were introduced in $5 \mathrm{~mL}$ of THF and the mixture was stirred overnight. It was then centrifuged at $60000 \mathrm{rpm}$ for $60 \mathrm{~min}$ to separate the clay particles from the polymer chains contained in the supernatant. The supernatant solution was collected and dried overnight at room temperature. Attenuated total reflection Fourier transform infrared spectroscopy (ATR-FTIR) confirmed the absence of Laponite indicating an efficient 
separation while gravimetric analysis of the supernatant showed that more than $90 \%$ of the total amount of polymer formed could be extracted by this method. The recovered polymer chains were analyzed by size exclusion chromatography (SEC).

Analytical Techniques. ${ }^{1} \mathrm{H}$ NMR analysis was performed in $\mathrm{CDCl}_{3}$ at room temperature (Bruker DRX 300). Monomer consumption was followed by gravimetric analysis. SEC measurements were performed using a Viscotek TDAmax system from Malvern Instruments consisting of an integrated solvent and sample delivery module (GPCmax), a four-capillary differential viscometer, a differential refractive index detector (RI), and a diode array UV detector. Analyses were performed at $40{ }^{\circ} \mathrm{C}$ with $\mathrm{THF}$ as eluent at a flow rate of $1 \mathrm{~mL} \mathrm{~min}{ }^{-1}$, using toluene as a flow rate marker. All polymers were analyzed at the concentration of $5 \mathrm{mg}$ $\mathrm{mL}^{-1}$ after filtration through a $0.45 \mu \mathrm{m}$ pore-size membrane. The separation was carried out on three Polymer Laboratories columns [3 x PLgel $5 \mu \mathrm{m}$ Mixed C $\left.\left(300 \times 7.5 \mathrm{~mm}^{2}\right)\right]$ and a guard column (PL gel $5 \mu \mathrm{m}$ )]. The average molar masses (number-average molar mass, $M_{\mathrm{n}}$, and weight-average molar mass, $\left.M_{\mathrm{w}}\right)$ and the molar mass dispersity $\left(~ Ð=M_{\mathrm{w}} / M_{\mathrm{n}}\right)$ were derived from the RI signal by a calibration curve based on polystyrene standards (PS from Polymer Laboratories). FTIR spectra were recorded on a Nicolet FTIR 460 spectrometer using the ATR mode. The spectra were obtained at a resolution of $4.0 \mathrm{~cm}^{-1}$ at room temperature in a wavenumber range between 4000 and $400 \mathrm{~cm}^{-1}$.

Average hydrodynamic diameters $\left(\mathrm{Z}_{\mathrm{av}}\right)$ were measured by photon correlation spectroscopy at $90^{\circ}$ (Zetasizer $1000 \mathrm{HSA}$ from Malvern). At least five measurements were performed for each sample. The broadness of the distribution was given by a dimensionless number called Poly (the higher this value, the broader the size distribution). Samples for transmission electron microscopy (TEM) analyses were dropped on a carbon-coated copper grid and dried under air. The TEM images were recorded using a Philips CM120 transmission electron 
microscope at an accelerating voltage of $80 \mathrm{kV}$ (Centre Technologique des Microstructures $(\mathrm{CT} \mu)$, plateforme de l'Université Claude Bernard Lyon 1, Villeurbanne, France). Ultrathin sections $(50-100 \mathrm{~nm})$ were obtained at room temperature, after embedding the composite particles in an epoxy resin using a Leica Ultracut E ultramicrotome equipped with a diamond knife as reported elsewhere. ${ }^{40}$

\section{RESULTS AND DISCUSSION}

\section{PEO2K-TTC adsorption onto Laponite}

Figure 1 shows the adsorption isotherm of PEO2 $K$-TTC onto Laponite at a fixed clay concentration of $10 \mathrm{~g} \mathrm{~L}^{-1}$ and $\mathrm{pH}=9.8$. Strong adsorption of PEO2K-TTC macromolecules onto Laponite platelets was observed at low concentration and the adsorbed amount then increased steadily with increasing the macroRAFT agent concentration. As expected from the L-type shape of the curve, the isotherm data fitted well to the Langmuir adsorption model (Figure SI-1, Supporting Information). The adsorbed amount at saturation determined using the Langmuir equation (i.e., $515 \mathrm{mg} \mathrm{g}^{-1}$ ) agrees well with the experimental data reported in the literature for the adsorption of $\mathrm{PEO} 2 \mathrm{~K}$ onto Laponite ${ }^{41}$ indicating that the thiocarbonyl thio end group of the macroRAFT agent has no noticeable effect on the adsorption. PEO2KTTC adsorption onto Laponite is of the high affinity type with a binding energy constant, $\mathrm{K}_{\mathrm{L}}$, of $5.8 \mathrm{~L} \mathrm{mg}^{-1}$ as determined from the linearized form of the equation. 


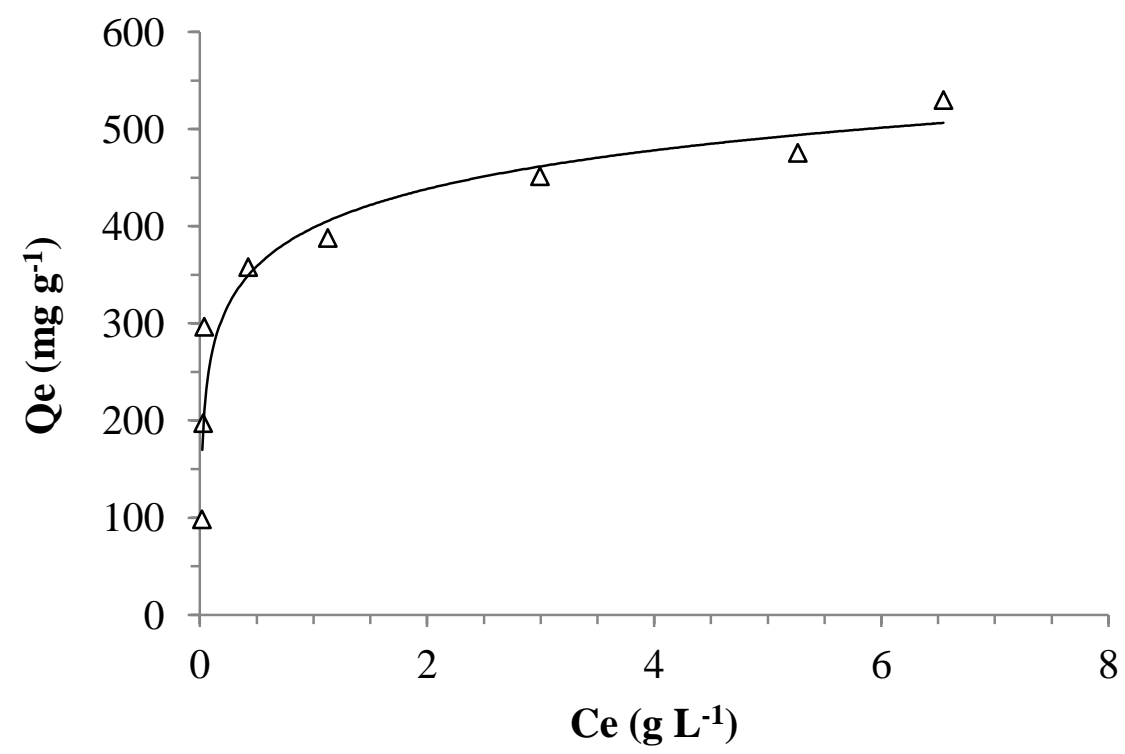

Figure 1. Adsorption isotherm of PEO $2 K$-TTC onto Laponite $\left(10 \mathrm{~g} \mathrm{~L}^{-1}\right)$. The experimental data are well fitted by the Langmuir adsorption model with a high correlation coefficient. The linearized form of the isotherm and the associated Langmuir constants are reported in the Supporting Information (Figure SI-1).

\section{Synthesis of Laponite/PS composite latexes by RAFT-mediated emulsion} polymerization

Two first experiments were carried out in emulsion, both mediated by the macroRAFT agent. The first one was an ab initio emulsion polymerization of styrene (run 1) while the second one was an experiment performed under the same conditions but in the presence of 5 wt\% of Laponite based on monomer amount (run 2). When the polymerization was carried out in the absence of Laponite, a low polymerization rate with a long induction period (ca. 2h) was observed, the final conversion leveling off at around $35 \%$ after 24 hours (Figures $2 \mathrm{a}$, b). In addition, the macroRAFT agent was not capable to efficiently stabilize the latex particles as attested by the large particle size $\left(\mathrm{Z}_{\mathrm{av} .}>1 \mu \mathrm{m}\right.$, Table 1$)$ and the Poly value $(0.7)$ 
which both suggested particle aggregation. These findings were consistent with previous studies employing PEO-based macroRAFT agents in emulsion polymerization of styrene. ${ }^{25}$ Indeed, a similar PEO macroRAFT was shown to partition between the monomer droplets and the aqueous phase ${ }^{28}$ impeding a fast growth of the PS blocks and thus an efficient nucleation. In contrast, the polymerization carried out in the presence of Laponite (run 2) was faster with a short induction period (ca. 1h) and $74 \%$ of conversion being achieved after $7 \mathrm{~h}$ of reaction. Similar results were obtained for experiments conducted with lower PEO2K-TTC concentrations and a fixed clay content resulting in lower amounts of free (i.e., non adsorbed) macroRAFT in water (runs 3 and 4, respectively, Table 1). In the absence of inorganic component, this induction period generally corresponds to the first chain transfer reactions required for the PS block to reach a sufficient molar mass to become surface active and selfassemble in water (nucleation). Here, consistently, the higher the concentration of free PEO macroRAFT in water (Ce in Table 1), the longer the induction period (Figure 2b). In run 2, stable particles were formed with an average diameter of $287 \mathrm{~nm}$ and a Poly value of 0.2 (Table 1). The improved colloidal stability of the polymer/clay composite particles compared to the pure PS latex may be rationalized by the following. PEO2 $K$-TTC adsorption on the clay surface decreases the probability of macroRAFT agent diffusion into the monomer phase, which would guarantee that most of the macroRAFT agent macromolecules are available to allow their chain extension with styrene. In addition, the adsorbed macroRAFT ensures rapid localization of the polymerization at the surface of the platelets, which would promote the efficient nucleation of hybrid particles stabilized by the Laponite/macroRAFT complex. Both effects would contribute to a more efficient use of the macroRAFT agent at the polymerization locus, and hence to a better nucleation and stabilization efficiency. 
$\mathbf{a}$

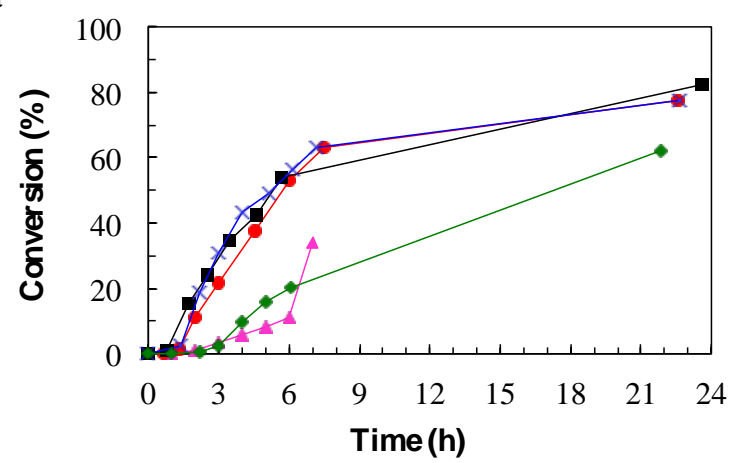

c

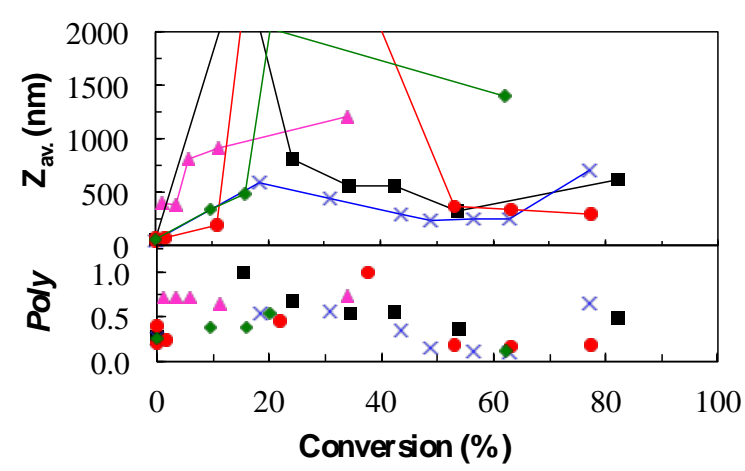

b

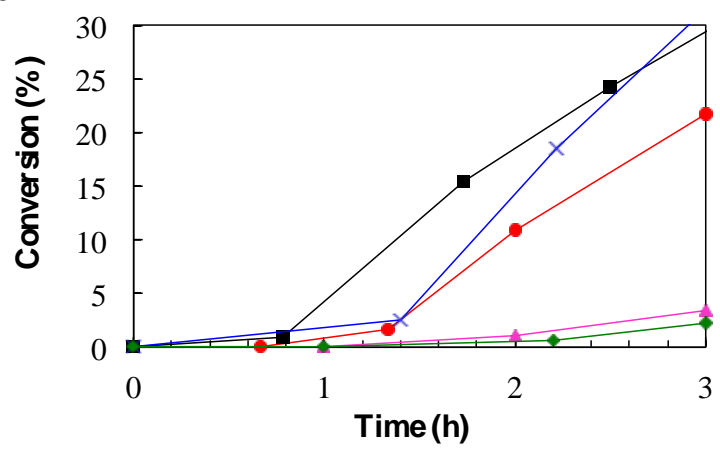

- No Clay $\quad 4.4 \mathrm{mM}$ PEO2K-TTC (run 1)

- 5 wt\% Clay $4.4 \mathrm{mM}$ PEO2K-TTC (run 2)

- 5 wt $\%$ Clay 2.2 mM PEO2K-TTC (run 3)

* 5 wt $\%$ Clay 3.3 mM PEO2K-TTC (run 4)

$\bullet 5$ wt $\%$ Clay 8.8 mM PEO2K-TTC (run 5)

Figure 2. Evolution of monomer conversion with time $(a, b)$ and of the average particle diameter and Poly value with conversion (c) during the synthesis of pure PS and Laponite/PS nanocomposite particles via RAFT-mediated emulsion polymerization using increasing concentrations of PEO2 $K$-TTC macroRAFT agent and a constant concentration of ACPA (3.5 mM). [Laponite] $=5 \mathrm{wt} \%$ based on styrene.

Figure 2c shows the effect of the macroRAFT concentration on the evolution of particle diameters with conversion for a fixed clay content of $5 \mathrm{wt} \%$. Polymerizations conducted with low PEO2K-TTC concentrations (i.e., 2.2, 3.3 and $4.4 \mathrm{mM}$, runs 3, 4 and 2, respectively) exhibited similar size evolutions. This indicates that in the presence of clay, the particle nucleation is similar and rather governed by the clay, with a peculiar behavior - that will be commented below - during the first $40 \%$ conversion for which a transitory destabilization period is observed. However, increasing the PEO2K-TTC concentration to $8.8 \mathrm{mM}$ (run 5) resulted in a poor colloidal stability similar to the one observed in the absence of clay (Table 
1). Besides, this was accompanied by a decrease of the reaction rate preceded by a long induction period as previously discussed. As seen in Table 1 and as mentioned earlier, the amount of free un-adsorbed $\mathrm{PEO} 2 K$-TTC increased with increasing the macroRAFT concentration. For run 5, this amount corresponded to $71 \mathrm{~mol} \%$ of the total amount of macroRAFT agent introduced. The free macroRAFT agent may thus diffuse in the monomer phase, which would have undesirable effects similar to those previously mentioned for the polymerization performed in the absence of clay (run 1), notably in terms of particle size which is very large (Table 1).

Despite the substantial improvement in terms of colloidal stability and kinetics for macroRAFT agent concentrations close to or lower than typically $4.4 \mathrm{mM}$, all reactions carried out in the presence of Laponite exhibited a transient instability characterized by a limited coagulation up to around $50 \%$ conversion after which the system became stable again. These results are similar to those previously reported by our group ${ }^{42,43}$ and others ${ }^{44}$ during the synthesis of Laponite-armored latexes by surfactant-free emulsion polymerization. This temporary aggregation phenomenon can be attributed to the presence of clay platelets that tend to adsorb on monomer droplets and decrease consequently the amount of clay available for stabilization of the aqueous phase nucleated latex particles. The system then regained stability at high conversions upon the release of clay platelets from the depleted monomer droplets. In line with this temporary coagulation phenomenon, the formed aggregates disappeared after around 30\% conversion as colloidal stability was recuperated, resulting in a drop of the particles size, as expected. In order to confirm this hypothesis, we performed an experiment (run 6, Table 1) for which only a small fraction of styrene was introduced at the beginning of the reaction and the rest slowly fed to minimize the presence of monomer droplets. As shown in Figure 3, there was no longer temporary aggregation phenomenon 
occurring under these conditions compared to the same experiment performed in batch (run 7 in Table 1).

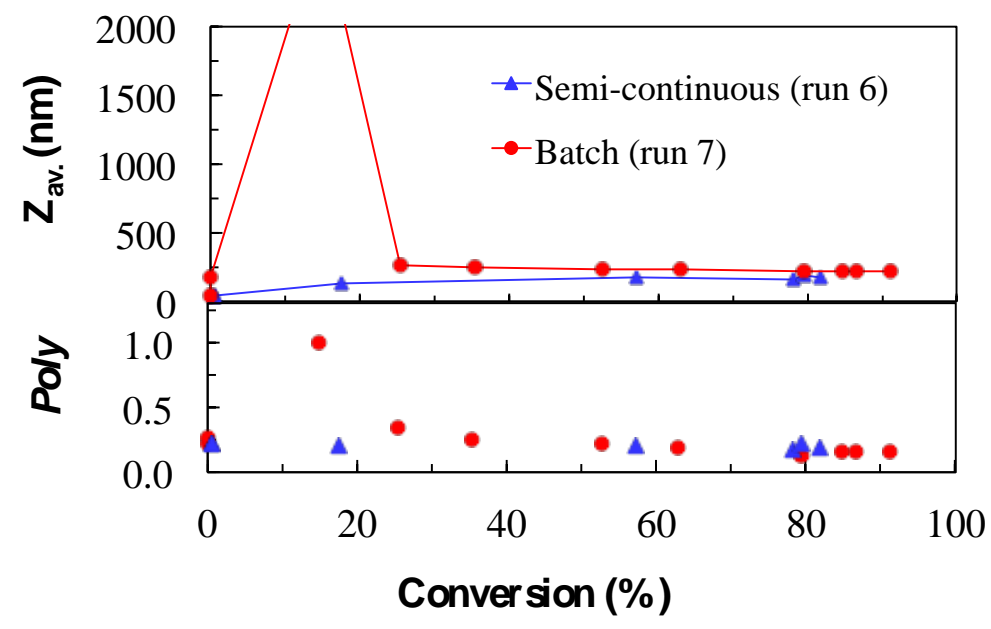

Figure 3. Evolution of the average particle diameter and Poly value with conversion during the synthesis of Laponite/PS nanocomposite latexes via RAFT-mediated emulsion polymerization using PEO2 $K$-TTC as macroRAFT agent using a semi-continuous (run 6) or a batch (run 7) process.

Regarding the complexity of the system (notably the partitioning of PEO and PEO/Laponite complex), the mechanisms leading to the formation of the particles may be quite complicated in the present case and are not completely elucidated. Based on the previous observations, one may however make the following assumptions. The aforementioned observation that induction periods are dependent on the amount of free PEO macroRAFT agent in water would suggest that the chain extension of the PEO macroRAFT agents in water is the main event governing the kinetics. The nucleation would then start by the self-assembly of PEO- $b$-PS amphiphilic block copolymers generating particles that quickly adsorb onto PEO macroRAFT/clay complex dispersed in water (Figure 4A). Obviously, polymer chains may also grow concurrently on the clay surface, which would favor subsequent block copolymers adsorption. The polymerization would then mainly occur at the surface of the clays and the resulting nuclei (i.e. the macroRAFT/clay complex and the 
self-assembled block copolymers) could then aggregate to form particles stabilized by clay platelets and adsorbed PEO segments (Figure 4A).
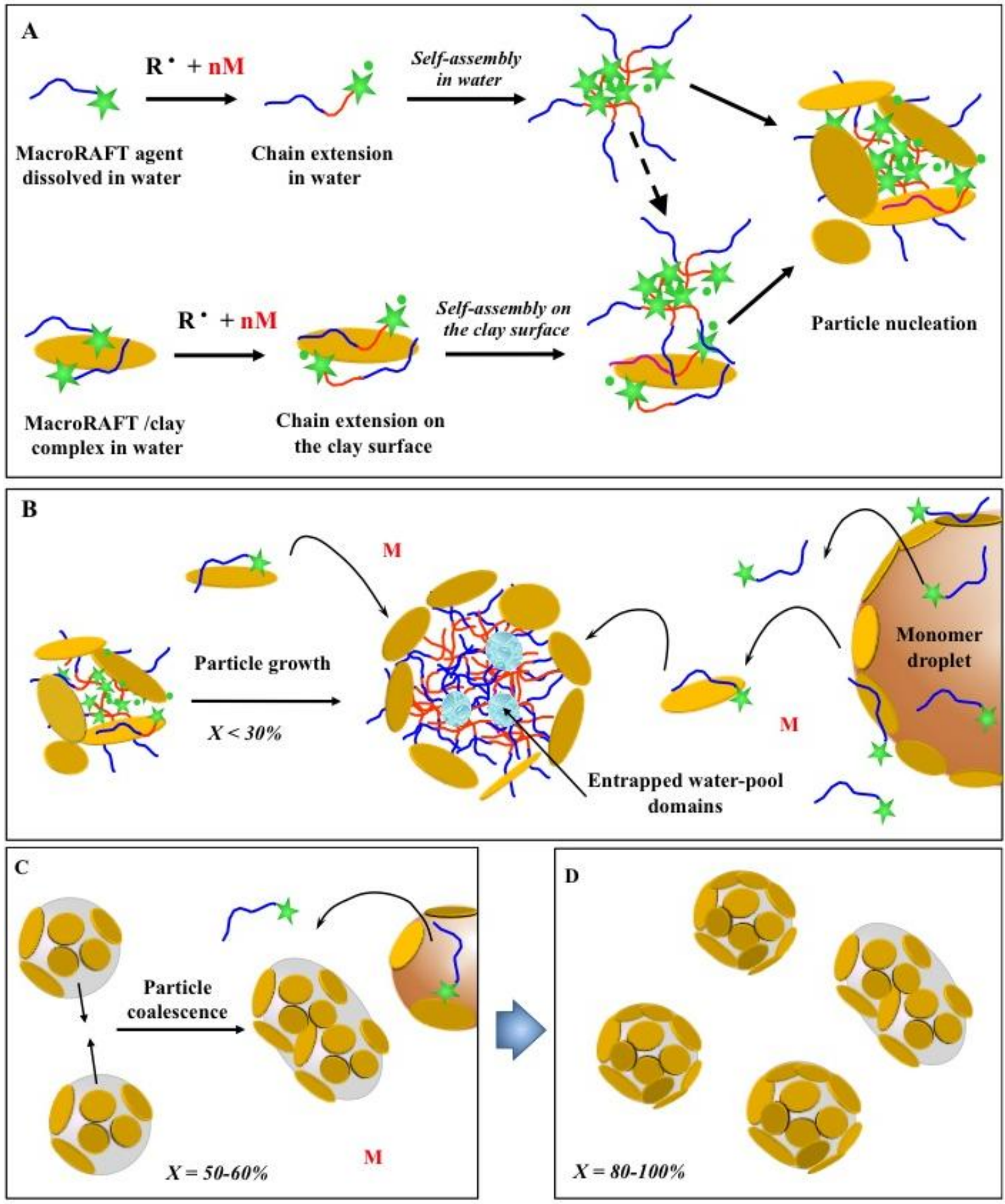

Figure 4. Scheme illustrating the mechanism of formation of Laponite-armored multi-hollow latexes by RAFT-mediated surfactant-free emulsion polymerization. A) Particle nucleation. B, C and D) Particle growth. 
Upon growing, the particles would become more and more hydrophobic and thus necessitate more clay platelets and/or PEO to maintain colloidal stability (Figure 4B). The free PEO that is initially in the monomer droplets or adsorbed on the clay platelets at the droplet/water interface would likely participate to the stabilization of the particles by forming, with the disappearance of the monomer droplets, PEO- $b$-PS block copolymers (alone or together with the clay platelets). Alternatively, the system can also gain stability through heterocoagulation with block copolymer/clay complexes present in water or through coagulation with another growing particle in order to increase the surface coverage of the final particles with the Laponite discs and minimize interfacial free energy (Figure 4C). As the clay is hydrophilic, it has a strong tendency to be located at the surface of the particles forming an armored morphology although block copolymers chains may have initially grown from each side of the clay sheets. In this process, some PEO2 $K$-TTC macroRAFT agent or PEO- $b$-PS block copolymers may also become embedded in the composite particles and entrap water with them, thereby generating multi-hollow particles decorated with Laponite platelets as will be further demonstrated in section 4 below.

\section{Evidence of the formation of PEO-b-PS amphiphilic block copolymers}

The positive results obtained in the presence of Laponite in terms of colloidal stability and kinetics led us to investigate further the controlled character of the growing polymer chains by determining their molar mass and molar mass distribution by SEC. Figure 5a shows the shift of the SEC traces with conversion for the polymer synthesized via ab initio emulsion polymerization in the absence of clay (run 1). Despite the linear evolution of $M_{\mathrm{n}}$ with conversion (Figure 6), the broad molar mass distribution (at least two populations can be identified in the chromatograms) indicates a poor control of the polymerization. As already 
mentioned, this is likely related to PEO2K-TTC partitioning between the monomer droplets and the aqueous phase resulting in different molar mass populations as reported previously. ${ }^{28}$
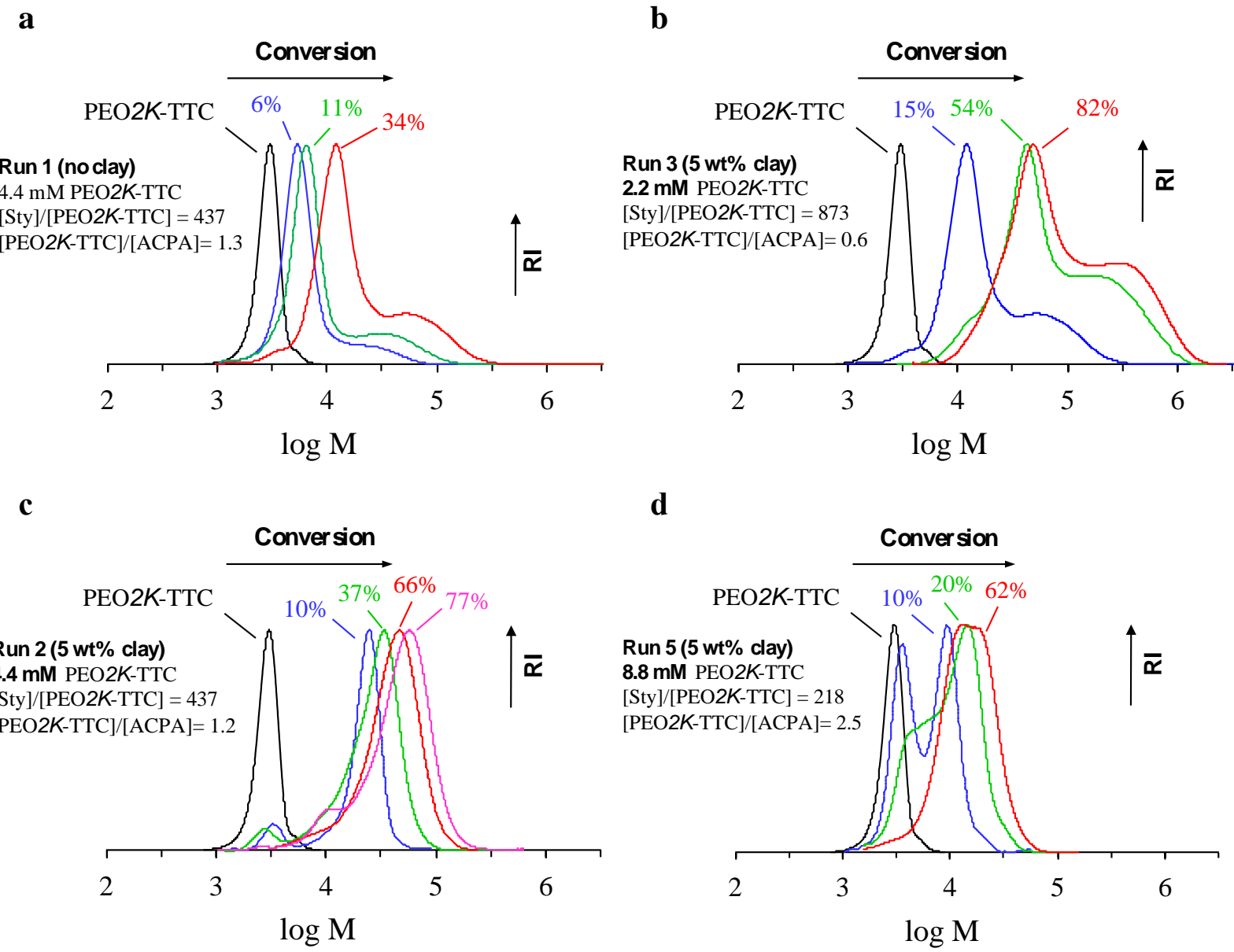

e

\section{f}
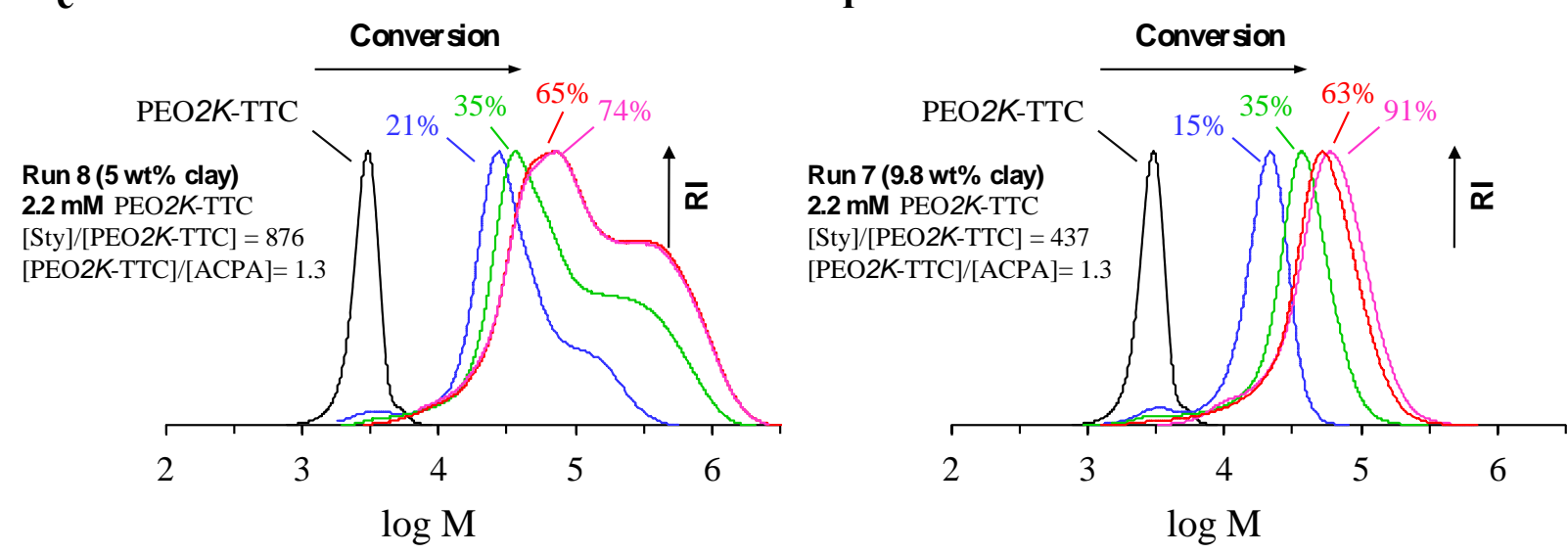

Figure 5. Evolution of the size exclusion chromatograms with monomer conversion (SEC

THF, PS calibration) during the synthesis of PS and Laponite/PS nanocomposite latexes by RAFT mediated emulsion polymerization of styrene. a), b), c) and d) Effect of PEO2K-TTC concentration for a constant concentration of ACPA (3.5 mM). e) Effect of ACPA 
concentration for $[\mathrm{PEO} 2 \mathrm{~K}$-TTC] $=2.2 \mathrm{mM}$ and $\mathrm{f}$ ) Effect of styrene concentration for [ACPA] $=1.6 \mathrm{mM}$ and $[\mathrm{PEO} 2 K-\mathrm{TTC}]=2.2 \mathrm{mM}$.

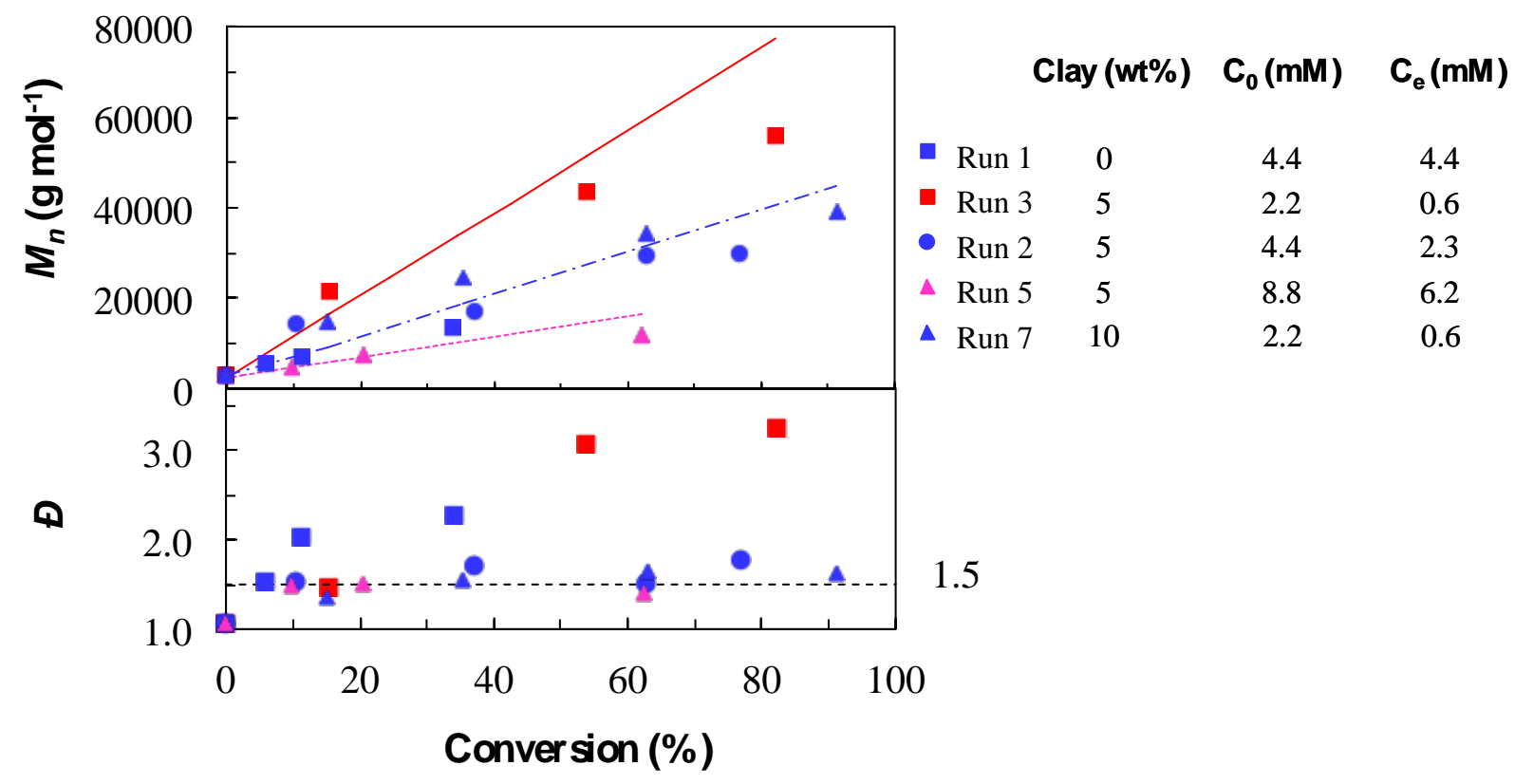

Figure 6. Effect of PEO2 $K$-TTC macroRAFT agent concentration on the evolution of the number-average molar masses $\left(M_{\mathrm{n}}\right)$ and the molar mass distributions $(\nexists)$ with monomer conversion during the synthesis of pure PS and Laponite/PS nanocomposite latexes via RAFT-mediated surfactant-free emulsion polymerization (runs 1, 2, 3 and 5). Run 7 illustrates the effect of styrene concentration for $[\mathrm{PEO} 2 K$-TTC] $=2.2 \mathrm{mM}$. The straight lines in the $M_{\mathrm{n}}$ versus conversion plots correspond to the theoretical evolutions (equation 2).

run $3,(\ldots .$.$) runs 1,2$ and 7 and (_---.) run 5.

For the polymerizations carried out in the presence of clay (runs 2 and 3), after $10 \%$ and $15 \%$ of conversion, a very nice shift of the chromatograms is observed with almost a complete consumption of the starting PEO macroRAFT agent. This is in agreement with the early formation of well-defined PEO- $b$-PS amphiphilic block copolymers in water $(\nexists=1.5$ for both experiments). When the conversion increased, the control of the growth of the block 
copolymer is kept for run $2(\bigoplus=1.6$ after $77 \%$ conversion $)$, while the molar mass distribution broadened and a second population of high and uncontrolled molar mass appeared for run 3, probably due the low ratio of $\mathrm{PEO} 2 K$-TTC/ACPA employed in this case (0.6). As a consequence, the experimental and theoretical number-average molar masses were in poor agreement (Figure 6). As the amount of adsorbed PEO2K-TTC was not that different (1.63 $\mathrm{mM}$ for run 3 and $2.06 \mathrm{mM}$ for run 2), this would indicate that the ratio [free macroRAFT]/[ACPA] would actually play an important role in the control of the polymerization. This again highlights the predominance of the events occurring in the water phase with the free macroRAFT. For run 2, part of PEO2 $K$-TTC is consumed slowly but there is no residual macroRAFT for conversion higher than $37 \%$. This slowly consumed fraction of PEO2 $K$-TTC very likely corresponds to the fraction of the macroRAFT that is not adsorbed onto Laponite and that may partition between the aqueous phase and the monomer droplets. Besides, as mentioned above there is also probably a low amount of the PEO chains adsorbed onto Laponite particles present on the monomer droplets. These two populations of PEO (free and adsorbed onto Laponite particles not initially dispersed in water) would be slowly consumed in the medium particularly with the disappearance of the monomer droplets. This would explain the appearance of a shoulder on the low molar mass side of the distributions for high conversion (63 and 77\%, Figure 5c). Consequently, the experimental number-average molar masses were higher than predicted at low conversions $(<40 \%)$, but matched quite well the theoretical values at higher conversions. Indeed, as explained above, a fraction of PEO2 $K$-TTC is not able to participate to the RAFT polymerization at the beginning of the reaction. As the polymerization proceeds, the reactivation of the PEO2KTTC and formation of new PEO- $b$-PS block copolymers lead to experimental values closer to the theoretical ones. A similar behavior was observed for the reaction carried out with 8.8 $\mathrm{mM}$ of PEO2K-TTC (run 5, Figure 5d) with, however, a more pronounced effect of the free 
PEO2K-TTC for this polymerization. Indeed, although in this case the experimental numberaverage molar masses agreed quite well with the theoretical ones (Figure 6), higher amounts of residual PEO2K-TTC were observed in the SEC traces at low conversion $(<20 \%)$, which is in agreement with the greater amount of free PEO2K-TTC in the aqueous phase (i.e. 6.24 $\mathrm{mM}$, three times more than in the previous case for approximately the same amount of adsorbed PEO2K-TTC, Table 1).

The next experiment was performed with a lower amount of ACPA to reach the same initial ratio $[\mathrm{PEO} 2 K-\mathrm{TTC}] /[\mathrm{ACPA}]$ than in run 2 (run 8, Table 1) while maintaining a low amount of free macroRAFT. Unfortunately, SEC analysis again revealed a broad molar mass distribution (Figure 5e). In a last attempt to improve the living character of the polymerization, styrene concentration was divided by two while maintaining the same $[$ Sty]/[PEO2K-TTC] and [PEO2K-TTC]/[ACPA] ratios as for run 2 (run 7, Table 1). This resulted in a good control of the polymerization with narrow molar mass distribution (Figure $5 f, Ð=1.6$ for the final latex), a nice fit of the theoretical values by the experimental data (Figure 6) and almost no effect of the free PEO chains. The initial amount of styrene obviously determines the partitioning of free PEO2K-TTC: the same initial amount of free macroRAFT (run 7 vs run 8) would not result in the same repartition between water and the monomer droplets. According to the SEC results, a lower amount of monomer would result in less perturbation of the polymerization by the PEO chains that are released when these droplets disappear.

\section{Particle morphology}

The particles obtained during the polymerization of run 7 have been imaged by TEM. Figure 7 reveals the formation of composite latexes exhibiting inside holes, according to the 
so-called multi-hollow morphology. ${ }^{45,46}$ Similar morphologies were observed ${ }^{27}$ when using an amphiphilic PEO-based trithiocarbonate chain transfer agent in the emulsion copolymerization of MMA and BA. Multi-hollow particles were also observed during the RAFT-mediated miniemulsion polymerization of styrene using a PEO-based macroRAFT agent carrying a dithiobenzoate terminal end group. ${ }^{28}$ This particular morphology was due to the incorporation of hydrophilic PEO chains inside the hybrid particles either as homopolymers at the beginning of the polymerization as a result of macroRAFT partitioning, or as segments of PEO- $b$-PS diblock copolymers formed during the polymerization. The entrapped PEO chains are draining water molecules forming nanosized water-pool domains. These domains resulted in holes after drying the sample for TEM and appeared as small light grey spots. In our case, these "holes" were observed for samples withdrawn at the beginning of the polymerization indicating that they were formed in the very early stages of the polymerization in perfect agreement with the nucleation mechanism depicted in Figure 4, and involving (i) the formation of block copolymers, (ii) their adsorption onto PEO2 $K$-TTC/clay complexes, and (iii) the further polymerization inside the resulting aggregated objects. The Laponite platelets could not be clearly seen in the TEM images, probably due to the high electronic density of polystyrene that reduces the contrast between the organic and inorganic phases. However, the irregular particles shape supports the hypothesis of formation of clayarmored latexes. To overcome this limitation, which is typical of TEM analyses, the composite particles were embedded in an epoxy resin and microtomed into ultra-thin slices before TEM observation. The TEM images of Figure 8 reveal a honeycomb-like nanostructure, indicating that the Laponite platelets were located at the latex particle surface forming the so-called clay-armored morphology in agreement with the proposed mechanism. To the best of our knowledge, this is the first time that such multi-hollow clay-armored particles are reported in the literature. 

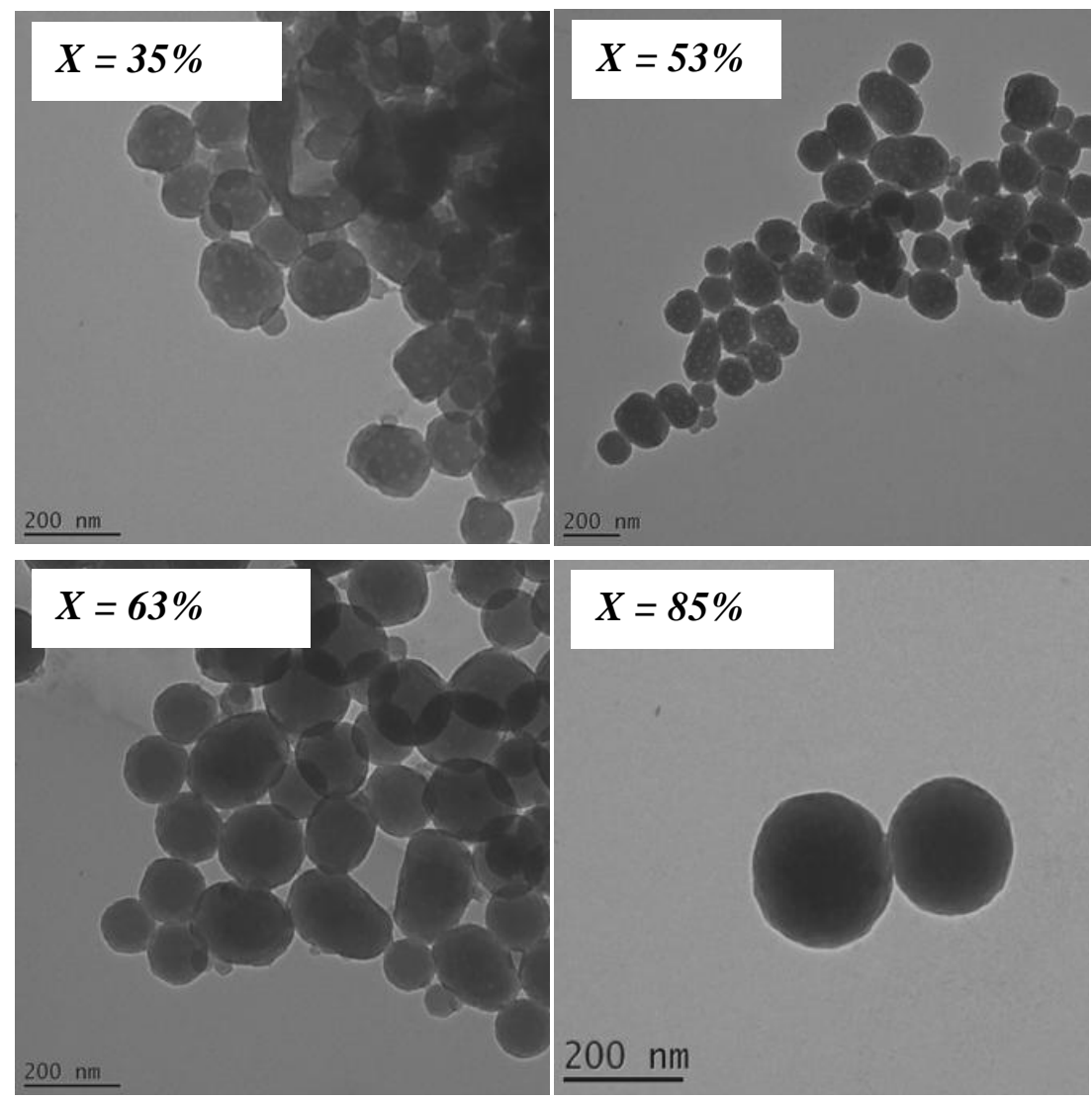

Figure 7. TEM images of Laponite/PS composite latex particles (run 7, Table 1) for increasing monomer-to-polymer conversions.
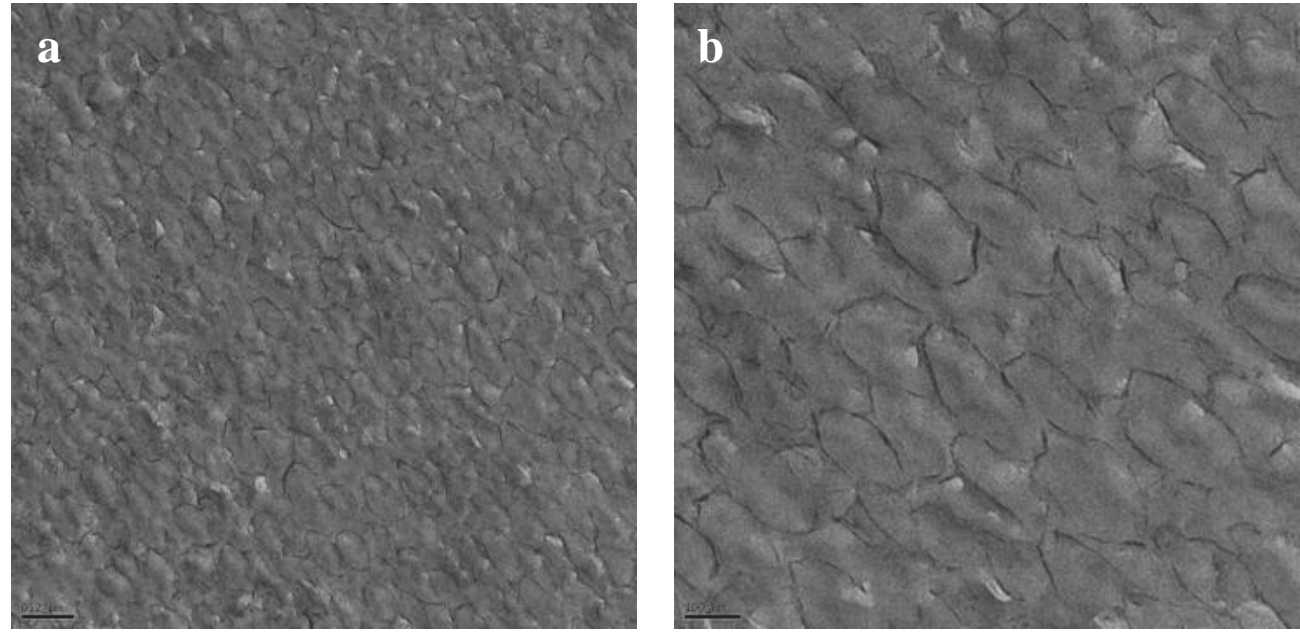

Figure 8. TEM images at intermediate (a) and high (b) magnifications of an ultrathin crosssection of epoxy-embedded Laponite/PS composite latex particles (run 7, Table 1). 


\section{Extrapolation to the emulsion copolymerization of MMA and BA}

This section aims at demonstrating that the RAFT-based strategy investigated in this paper is not limited to styrene but can also be successfully extrapolated to other monomers enlarging the potential range of applications of the so-formed clay-armored latexes. Hence, the RAFT-mediated emulsion polymerization of MMA and BA $(80: 20 \mathrm{v} / \mathrm{v})$ in the presence of $6 \mathrm{wt} \%$ Laponite (run 9, Table 1) was briefly investigated in a semi-continuous process. The initial macroRAFT concentration was fixed to $2.5 \mathrm{mM}\left([\mathrm{PEO} 2 K-\mathrm{TTC}]_{\text {free }}=0.79 \mathrm{mM}\right)$ and the $[$ monomers $] /[\mathrm{PEO} 2 K$-TTC] ratio to 355 (overall solids content $=9.5 \mathrm{wt} \%$ ), experimental conditions which are close to those used for run 6. A stable coagulum-free latex $\left(Z_{\mathrm{av} .}=264\right.$ nm, Poly $=0.2)$ was obtained after ca. 7 hours, which corresponds to $100 \%$ monomer conversion (Figure SI-2a in the Supporting Information). The SEC chromatograms are shifted towards higher molar masses with conversion (Figure 9a) and the molar mass distribution remains quite narrow $(\nexists=1.6$, Table 1$)$. In addition all experimental $M_{\mathrm{n}}$ data points fall close to the theoretical line (Figure $9 \mathrm{~b}$ ). These results indicate that the polymerization is effectively under RAFT control. Attempts to polymerize MMA/BA mixtures in the sole presence of Laponite platelets in a conventional (i.e., non-controlled) soap-free emulsion polymerization process (so-called Pickering emulsion polymerization) were unsuccessful for MMA/BA weight ratios larger than typically $0.67 .{ }^{44}$ Although the exact reason for this failure remains unclear, the fact that we could successfully polymerize a MMA-rich comonomer composition through RAFT-mediated emulsion polymerization not only illustrates the potential of the RAFT technique in the synthesis of clay-armored latexes but also highlights the complexity of the mechanism of particles formation and stabilization of such systems. 


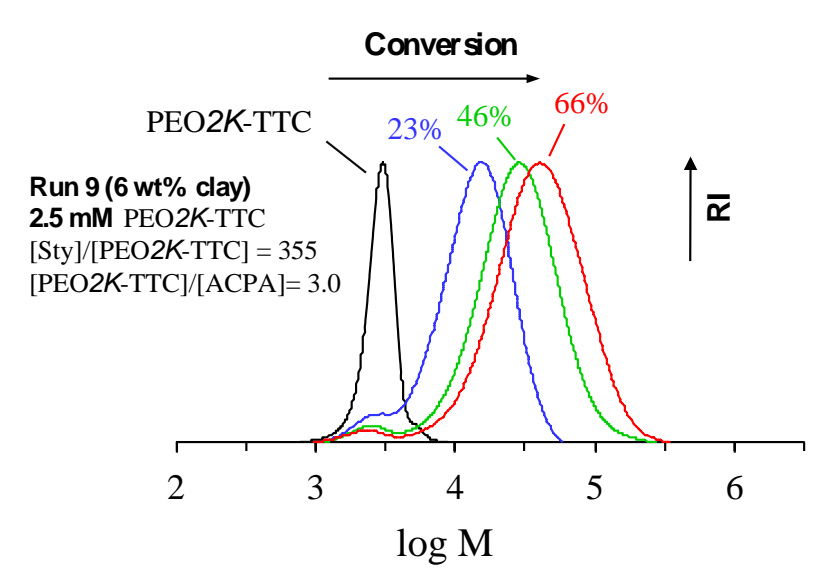

b

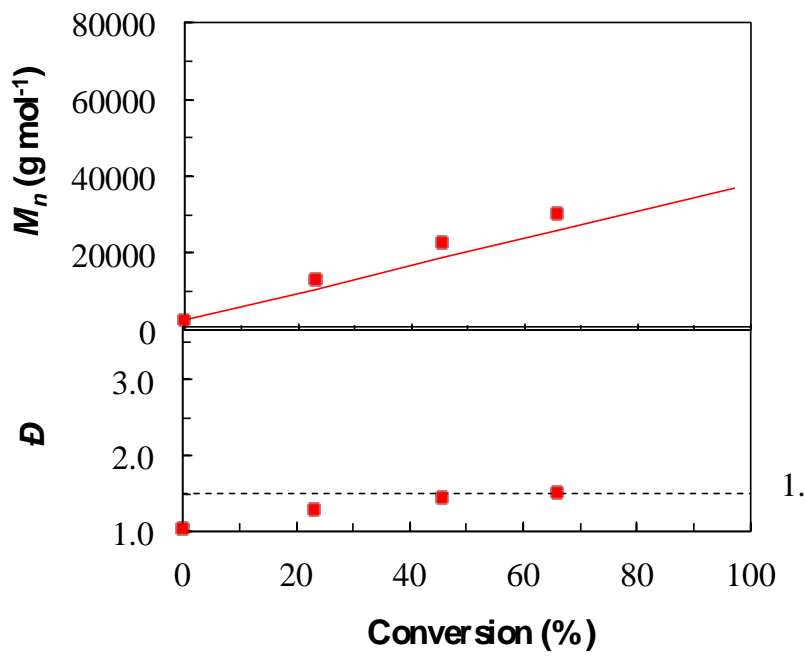

Figure 9. Evolution of a) the size exclusion chromatograms and b) the number-average molar masses $\left(M_{n}\right)$ and the molar mass distributions $(\bigoplus)$ with monomer conversion (SEC THF, PS calibration) during the synthesis of Laponite/P(MMA-co-BA) nanocomposite latexes by RAFT-mediated emulsion polymerization using PEO2K-TTC as macroRAFT agent.

\section{CONCLUSIONS}

Multi-hollow Laponite-armored PS and P(MMA-co-BA) latex particles have been synthesized by surfactant-free RAFT-mediated emulsion polymerization using PEO2 $K$-TTC as macroRAFT agent. The later was shown to adsorb on the Laponite surface. The equilibrium adsorption data fitted well to the Langmuir isotherm model in the whole range of concentration studied with a maximum adsorption capacity of $515 \mathrm{mg} \mathrm{g}^{-1}$ indicating high affinity of the macroRAFT towards Laponite. The anchored thiocarbonyl thio functionalities were further used to control the RAFT-mediated emulsion polymerization of hydrophobic monomers leading to in situ formation of amphiphilic block copolymers. The undesirable effects observed under the same conditions in the absence of clay, like rate retardation, long 
induction period and loss of colloidal stability were significantly reduced in the presence of clay. A minimum amount of macroRAFT was necessary to ensure a good control of the polymerization and a narrow molar mass distribution while a too large amount had similar effects as in the absence of clay due to partitioning of the free non-adsorbing PEO2 $K$-TTC macroRAFT. Optimization of both the macroRAFT and monomer concentrations allowed synthesizing stable latex particles with a good control of the molar mass and molar mass distribution. The composite latex particles exhibited an armored morphology with embedded PEO domains likely originating from heterocoagulation of the self-assembled block copolymers formed in the aqueous-phase with the ones nucleated on the Laponite clay discs. The strategy was successfully extrapolated to the copolymerization of MMA and BA enlarging thereby the spectrum of polymer composite latex particles that can be synthesized by this method.

\section{Supporting Information}

Linearized form of the PEO2K-TTC adsorption isotherm. Experimental conditions for the copolymerization of MMA and BA in presence of Laponite, and main characteristics of the resulting latex. Evolution of monomer conversion with time and of the average particle size and Poly value with conversion during the synthesis of $\mathrm{P}(\mathrm{MMA}-$ co-BA)/Laponite nanocomposite latexes via RAFT-mediated emulsion polymerization.

\section{Acknowledgements}

Thiago R. Guimarães thank the C2P2 laboratory for providing financial assistance during its undergraduate internship. Ana Cenacchi Pereira is greatly acknowledged for her help in 
TEM analyses. The authors would like to thank Malvern Instruments SA for technical support.

\section{References}

1 Georges, M. K.; Veregin, R. P. N.; Kazmaier, P. M.; Hamer, G. K. Macromolecules 1993, 26, 2987-2988.

2 Solomon, D.H.; Rizzardo, E.; Cacioli, P. US Patent 4581 429, 1985.

3 Hanemann, T.; Vinga Szabo, D. Materials 2010, 3, 3468-3517.

4 Pyun, J.; Matyjaszewski, K. Chem. Mater. 2001, 13, 3436-3448.

5 Kohn, C.; Morel, F.; Beyou, E.; Chaumont, P.; Bourgeat-Lami, E. Macromolecules 2007, $40,7464-7472$.

6 Radhakrishnan, B.; Ranjan, R.; Brittain, W. J. Soft Matter. 2006, 2, 386-396.

7 Tsujii, Y.; Ohno, K.; Yamamoto, S.; Goto, A.; Fukuda, T. Adv. Polym. Sci. 2006, 197, 145 .

8 Ghannam, L.; Parvole, J.; Laruelle, G.; Francois, J.; Billon, L. Polym. Int. 2006, 55, 11991207.

9 a) Bartholome, C.; Beyou, E.; Bourgeat-Lami, E.; Chaumont, P.; Zydowicz, N. Polymer 2005, 46, 8502-8510. b) Kohn, C.; Morel, F.; Beyou, E.; Bourgeat-Lami, E. Macromolecules 2007, 40, 7464-7472.

10 a) Perruchot, C.; Khan, M. A.; Kamitsi, A.; Armes, S. P.; von Werne, T.; Patten, T. E. Langmuir 2001, 17, 4479-4481. b) Chen, X.; Armes, S. P. Adv. Mater. 2003, 15, 15581561. c) Chen, X. Y.; Armes, S. P.; Greaves, S. J.; Watts, J. F. Langmuir 2004, 20, 587595. d) Vo, C. D.; Schmid, A.; Armes, S. P.; Skai, K.; Biggs, S. Langmuir 2007, 23, 408413.

11 Lattuada, M.; Hatton, T. A. Langmuir 2007, 23, 2158-2168. 
12 Hu, F.; Neoh, K. G.; Cen, L.; Kang, E. T. Biomacromolecules 2006, 7, 809-816.

13 Beija, M.; Marty, J-D.; Destarac, M. Prog. Polym. Sci., 2011, 36, 845-886.

14 Roghani-Mamaqani, H.; Haddadi-Asl, V.; Salami-Kalajahi, M. Polym. Rev. 2013, 52, 142188.

15 Delaittre, G.; D’Agosto, F.; Charleux, B. Adv. Polym. Sci. 2010, 233, 125-183.

16 a) Qiu, J.; Charleux, B.; Matyjaszewski, K. Prog. Polym. Sci. 2001, 26, 2083-2134. b) Save, M.; Guillaneuf, Y.; Guilbert, R. G. Aust. J. Chem. 2006, 59, 693-711.

17 Moad, G.; Solomon, D. H. The Chemistry of Free Radical Polymerization, 2nd edn 2006 (Elsevier: Amsterdam).

18 Barner-Kowollik, C.; Editor, Handbook of RAFT Polymerization. Wiley-VCH: Weinheim, 2008.

19 Moad, G.; Rizzardo, E.; Thang, S. H. Australian Journal of Chemistry 2012, 65, 9851076.

${ }^{20}$ Keddie, D. J.; Moad, G.; Rizzardo, E.; Thang, S. H. Macromolecules 2012, 45, 53215342.

21 Save, M.; Guillaneuf, Y.; Gilbert, R. G. Aust. J. Chem. 2006, 59, 693-711.

${ }^{22}$ McLeary, J. B.; Klumperman, B. Soft Matter 2006, 2, 45-53.

${ }^{23}$ Cunningham, M. F. Prog. Polym. Sci. 2008, 33, 365-398.

${ }^{24}$ Ferguson, C. J.; Hughes, R. J.; Nguyen, D.; Pham, B. T. T.; Hawkett, B. S.; Gilbert, R. G.; Serelis, A. K.; Such, C. H. Macromolecules 2002, 35, 9243-9245; Ferguson, C. J.; Hughes, R. J.; Nguyen, D.; Pham, B. T. T.; Gilbert, R. G.; Serelis, A. K.; Such, C. H.; Hawkett, B. S. Macromolecules 2005, 38, 2191-2204.

25 Dos Santos, A. M.; Pohn, J.; Lansalot, M.; D'Agosto, F. Macromol. Rapid Commun. 2007, $28,1325-1332$. 
${ }^{26}$ Rieger, J.; Stoffelbach, F.; Bui, C.; Alaimo, D.; Jérôme, C.; Charleux, B. Macromolecules 2008, 41, 4065-4068.

${ }^{27}$ Rieger, J.; Osterwinter, G.; Bui, C.; Stoffelbach, F.; Charleux, B. Macromolecules 2009, $42,5518-5525$.

${ }^{28}$ Dos Santos, A. M.; Le Bris, T.; Graillat, C.; D’Agosto, F.; Lansalot, M. Macromolecules 2009, 42, 946-956.

${ }^{29}$ Boursier, T.; Chaduc, I.; Rieger, J.; D'Agosto, F.; Lansalot, M.; Charleux, B. Macromolecules 2011, 2, 355-362.

${ }^{30}$ Samakande, A.; Sanderson, R. D.; Hartmann, P. C. J. Polym. Sci. Part A. Polym. Chem. 2008, 46, 7114-7126.

31 a) Nguyen, D.; Zondanos, H. S.; Farrugia, J. M.; Serelis, A. K.; Such, C.H.; Hawkett, B. S. Langmuir 2008, 24, 2140-2150. b) Nguyen, D.; Such, C.H.; Hawkett, B. S. J. Polym. Sci. Part A. Polym. Chem. 2012, 50, 346-352.

${ }^{32}$ Ali, S. I.; Heuts, J. P. A.; Hawkett, B. S.; van Herk, A. M. Langmuir 2009, 25, 1052310533.

${ }^{33}$ Nguyen, D.; Such, C.H.; Hawkett, B. S. J. Polym. Sci. Part A. Polym. Chem. 2013, 51, $250-257$.

${ }^{34}$ Zhong, W.; Nguendia Zeuna, J.; Claverie, J. J. Polym. Sci. Part A. Polymer Chem. 2012, $50,4403-4407$.

35 Zgheib, N.; Putaux, J.-L.; Thill, A.; Bourgeat-Lami, E.; D'Agosto, F.; Lansalot, M. Polym. Chem. 2013, 4, 607-614.

36 Parfitt, R. L.; Greenland, D. J. Clay Minerals 1970, 8, 305-315.

37 Laponite Technical Bulletin L104/90/A; Laporte Industries Ltd.: p.1, 1990.

38 Boursier, T.; Chaduc, I.; Rieger, J.; D’Agosto, F.; Lansalot, M.; Charleux, B. Polym. Chem. 2011, 2, 355-362. 
$39 \mathrm{Xu}, \mathrm{X}$; Smith, A. E.; Kirkland, S. E.; McCormick, C. L. Macromolecules 2008, 41, 8429-843

${ }^{40}$ Negrete-Herrera, N.; Putaux, J.-L.; David, L.; Haas, F. D.; Bourgeat-Lami, E. Macromol. Rapid Commun. 2007, 28, 1567-1573.

${ }^{41}$ Mongondry, P.; Nicolai, T.; Tassin, J-F. J. Colloid Interf. Sci. 2004, 275, 191-196.

${ }^{42}$ Bourgeat-Lami, E.; Guimarães, T. R.; Cenacchi-Pereira, A.-M.; Alves, G. M.; Moreira, J. C.; Putaux, J.-L.; Dos Santos, A. M. Macromol. Rapid Commun. 2010, 31, 1874-1880.

${ }^{43}$ Sheibat-Othman, N.; Cenacchi-Pereira, A.-M.; Dos Santos, A. M.; Bourgeat-Lami E. Polym. Sci. Part A. Polym. Chem. 2011, 49, 4771-4784.

44 Teixeira, R. F. A.; McKenzie, H. S.; Boyd, A. A.; Bon, S. A. F. Macromolecules 2011, 44, $7415-7422$

45 Okubo, M.; Kobayashi, H.; Matoba, T.; Oshima, Y. Langmuir 2006, 22, 8727-8731.

46 Kobayashi, H.; Miyanaga, E.; Okubo, M. Langmuir 2007, 23, 8703-8708. 